\title{
GaAs microcrystals selectively grown on silicon: Intrinsic carbon doping during chemical beam epitaxy with trimethylgallium
}

T. Molière, A. Jaffré, J. Alvarez, D. Mencaraglia, J. P. Connolly, L. Vincent, G. Hallais, D. Mangelinck, M. Descoins, D. Bouchier, and C. Renard

Citation: J. Appl. Phys. 121, 035704 (2017); doi: 10.1063/1.4974538

View online: $\mathrm{http}: / / d x . d o i . o r g / 10.1063 / 1.4974538$

View Table of Contents: http://aip.scitation.org/toc/jap/121/3

Published by the American Institute of Physics

\section{Articles you may be interested in}

Effects of two-mode transverse optical phonons in bulk wurtzite AIGaN on electronic mobility in AIGaN/GaN quantum wells

J. Appl. Phys. 121, 035703035703 (2017); 10.1063/1.4974448

Correlation between temperature dependence of Raman shifts and in-plane strains in an AIGaN/GaN stack J. Appl. Phys. 121, 035702035702 (2017); 10.1063/1.4974366

Comprehensive study on initial thermal oxidation of $\mathrm{GaN}(0001)$ surface and subsequent oxide growth in dry oxygen ambient

J. Appl. Phys. 121, 035303035303 (2017); 10.1063/1.4974458

Investigation of the light-extraction efficiency in $280 \mathrm{~nm}$ AIGaN-based light-emitting diodes having a highly transparent p-AIGaN layer

J. Appl. Phys. 121, 013105013105 (2017); 10.1063/1.4973493

\section{A|P $\left.\right|_{\text {Applied Physics }} ^{\text {Journal of }}$}

INTRODUCING INVITED PERSPECTIVES

Ultrafast magnetism and THz spintronics

Authors: Jakob Walowski and Markus Münzenberg 


\title{
GaAs microcrystals selectively grown on silicon: Intrinsic carbon doping during chemical beam epitaxy with trimethylgallium
}

\author{
T. Molière, ${ }^{1,2, a)}$ A. Jaffré, ${ }^{2}$ J. Alvarez, ${ }^{2}$ D. Mencaraglia, ${ }^{2}$ J. P. Connolly, ${ }^{2}$ L. Vincent, ${ }^{1}$ \\ G. Hallais, ${ }^{1}$ D. Mangelinck, ${ }^{3}$ M. Descoins, ${ }^{3}$ D. Bouchier, ${ }^{1}$ and C. Renard ${ }^{1}$ \\ ${ }^{1}$ Centre de Nanosciences et de Nanotechnologies, CNRS, Univ. Paris-Sud, Université Paris-Saclay, \\ C2N - Orsay, 91405 Orsay cedex, France \\ ${ }^{2}$ GeePs-Supelec, UMR CNRS 8507, Université Pierre et Marie Curie, Université Paris-Sud, \\ 11 rue Joliot Curie, Plateau de Moulon, 91192 Gif sur Yvette, France \\ ${ }^{3} I M 2 N P, C N R S / A i x$-Marseille Université, Faculté de saint Jérôme, Marseille, France
}

(Received 26 September 2016; accepted 10 January 2017; published online 20 January 2017)

\begin{abstract}
The monolithic integration of III-V semiconductors on silicon and particularly of GaAs has aroused great interest since the 1980s. Potential applications are legion, ranging from photovoltaics to high mobility channel transistors. By using a novel integration method, we have shown that it is possible to achieve heteroepitaxial integration of GaAs crystals (typical size $1 \mu \mathrm{m}$ ) on silicon without any structural defect such as antiphase domains, dislocations, or stress, usually reported for direct GaAs heteroepitaxy on silicon. However, concerning their electronic properties, conventional free carrier characterization methods are impractical due to the micrometric size of GaAs crystals. In order to evaluate the GaAs material quality for optoelectronic applications, a series of indirect analyses such as atom probe tomography, Raman spectroscopy, and micro-photoluminescence as a function of temperature were performed. These revealed a high content of partially electrically active carbon originating from the trimethylgallium used as the Ga precursor. Nevertheless, the very good homogeneity observed by this doping mechanism and the attractive properties of carbon as a dopant once controlled to a sufficient degree are a promising route to device doping. Published by AIP Publishing.

[http://dx.doi.org/10.1063/1.4974538]
\end{abstract}

\section{INTRODUCTION}

For thirty years, researchers have been attempting to integrate III-V semiconductors, in particular, GaAs on silicon, the purpose being to combine the advantage of the dominant and much researched silicon technology with the good optical and electrical properties of gallium arsenide. Many applications are expected such as photodetectors, optical interconnections, and lasers in innovative optoelectronic integrated circuits (OEICs), in high mobility channel transistors, and in multijunction solar cells. ${ }^{1-7}$

However, three major intrinsic problems have to be overcome: the lattice parameter mismatch (about 4\%) generating a high density of threading dislocations, the polar or non polar nature of, respectively, $\mathrm{GaAs}$ or $\mathrm{Si}$, and the difference of thermal expansion coefficient, which is at the origin of cracks for thick GaAs layers. ${ }^{8}$ Several approaches to these problems have been investigated to date, but until now, no one succeeded in achieving the defect-free and cost-effective integration of 2D continuous GaAs layers. One of these approaches, selective area epitaxy (SAE) of GaAs on patterned $\mathrm{Si}$, has shown significant improvements, yielding mostly defect-free epitaxial layers. However, the relaxation process occurring at the $\mathrm{GaAs} / \mathrm{Si}$ interface leads to a high density of misfit dislocations at levels, which are prohibitive for applications involving carrier transport through the interface area. We have recently demonstrated by a new method called ELTOn (for Epitaxial Lateral

a)timothee.moliere@gmail.com overgrowth on Tunnel Oxide from nano-seed) the defectand strain-free heteropitaxy of micrometric GaAs crystal on $\mathrm{Si}$, yielding an effective electrical contact of the epitaxial layer to the substrate. ${ }^{9}$ The ELTOn approach needs highly selective epitaxial growth of GaAs on silicon, as it is currently achieved by metalorganic chemical vapor deposition MOCVD or chemical beam epitaxy (CBE). In both cases, the most commonly used gaseous precursor for the Ga element is trimethylgallium (TMGa) in combination with arsine (AsH3) or tertiarybutylarsine (TBAs). Nonetheless, several studies have reported high doping levels attributed to the presence of carbon in homoepitaxial GaAs grown from TMGa. ${ }^{10-12}$ In addition, our own previous I(V) measurements ${ }^{9}$ were consistent with a p-type non-intentional doping possibly due to carbon incorporation. It is then of primary importance for applications to evaluate the concentration of carbon from TMGa and the resulting dopant level in ELTOn microcrystals.

Hall effect and capacitance-voltage measurements are the dedicated methods for measuring the type and concentration of electrical carriers. However, due to the micrometric size of GaAs ELTOn crystals, electrical characterization along these lines is very challenging. Therefore, we will attempt to trace back the carrier concentration by indirect and contactless methods. First, atom probe tomography (APT) was performed to inform us on the concentration of carbon. Then, the effective carrier concentration and type were deduced from Raman and photoluminescence spectroscopies that could be easily performed in routine inspection. 


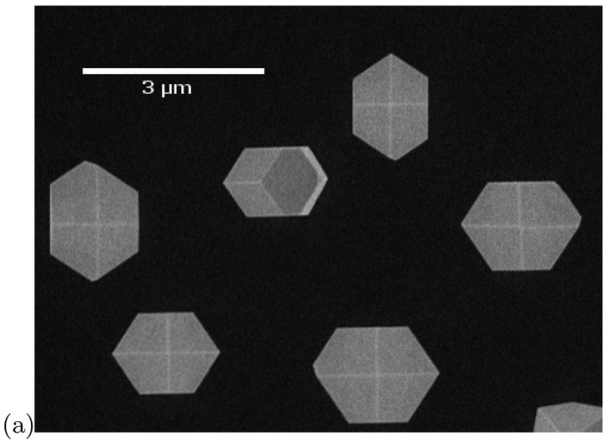

(b)

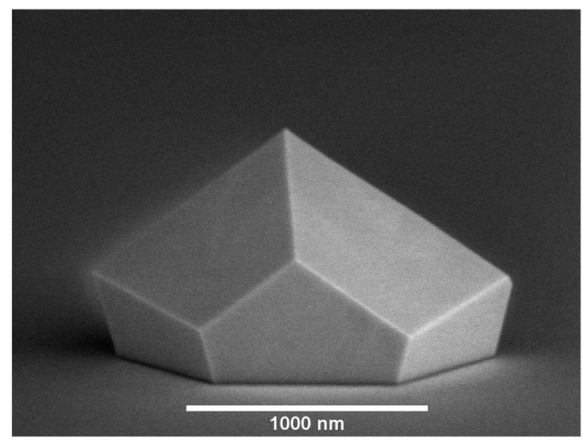

FIG. 1. GaAs/Si(100) epitaxial growth in planar view (a) and front view (b) by SEM.

\section{EXPERIMENTAL DETAILS}

The GaAs microcrystals were grown on silicon through a silica mask structured with nanoscaled openings (Fig. 1). The selective heteropitaxy was realized by chemical beam epitaxy (CBE) at the growth temperature of $575^{\circ} \mathrm{C}$ using the gaseous precursors Trimethylgallium (TMGa) and Tertiarybutylarsine (TBAs). For more details of the growth procedure, the reader is referred to the previous studies. ${ }^{9,13}$

APT analyses were performed using a Local Electrode Atom Probe (LEAP 3000X HR) at the IM2NP (Marseille, France) in the pulsed voltage mode. The specimen base temperature was about $20 \mathrm{~K}$. There were primarily extracted from GaAs microcrystals grown on silicon and prepared in nanometric tips by standard focalised-ion-beam (FIB) annular milling (Fig. 2).

The Raman spectra presented in this paper were obtained using a confocal microscope in a backscattering geometry. The injection and collection are achieved by the same lens. The Raman excitation source was a frequency-doubled Nd:YAG laser at $532 \mathrm{~nm}$ with an average power of $250 \mu \mathrm{W}$. A $50 \mu \mathrm{m}$ core diameter optical fiber acts as the confocal pinhole. The spot size around $1 \mu \mathrm{m}$ allowed the selective analysis of the unique GaAs microcrystal. A Si charge-coupled array detector and a 1800 grooves $/ \mathrm{mm}$ grating were used to record the spectra with a spectral resolution of $1 \mathrm{~cm}^{-1}$.

The micro-photoluminescence used the same configuration for the excitation source, whereas the detection is

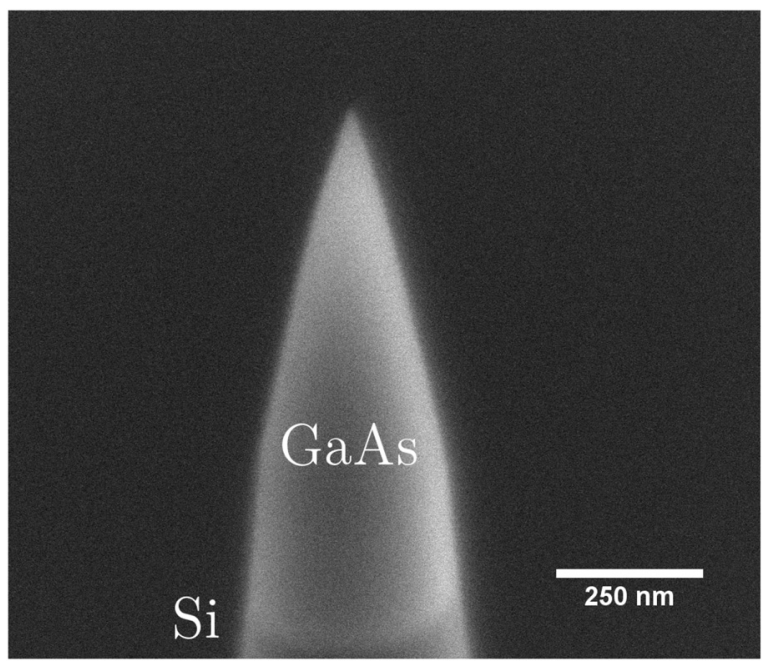

FIG. 2. The SEM image of a GaAs crystal prepared in nanometric tip by FIB. realized by a CCD InGaAs camera and a 150 grooves $/ \mathrm{mm}$ grating. The spot size is in this case $5 \mu \mathrm{m}$ due to the long range $20 \times$ infrared lens used. The temperature regulation was obtained by a nitrogen-based cryostat down to $80 \mathrm{~K}$. All the analyses were realized on a unique and representative crystal. Measurements performed on identical crystals have shown the same response.

\section{RESULTS AND DISCUSSION}

\section{A. Atom probe tomography}

The APT analysis was performed on two GaAs microcrystals grown on $\mathrm{Si}(100)$ at $575^{\circ} \mathrm{C}$ (Fig. 1) and $\mathrm{Si}(111)$ for comparison. The first one shows two twin boundaries, ${ }^{13}$ whereas the second one is perfectly monocrystalline and free of any structural defect. ${ }^{9}$

The quantification and spatial distribution of species based on the mass spectroscopy reveal that the GaAs is perfectly homogeneous (Fig. 3). Despite the first impression that might lead to believe that the concentration is higher at the interface (due to the varying cross section), 1D carbon concentration profiles performed throughout the tips did not reveal important density variation. Moreover, no significant difference was found between the two samples analyzed in terms of both atomic density and distribution showing that there is no precipitation of impurities at the twin boundaries.

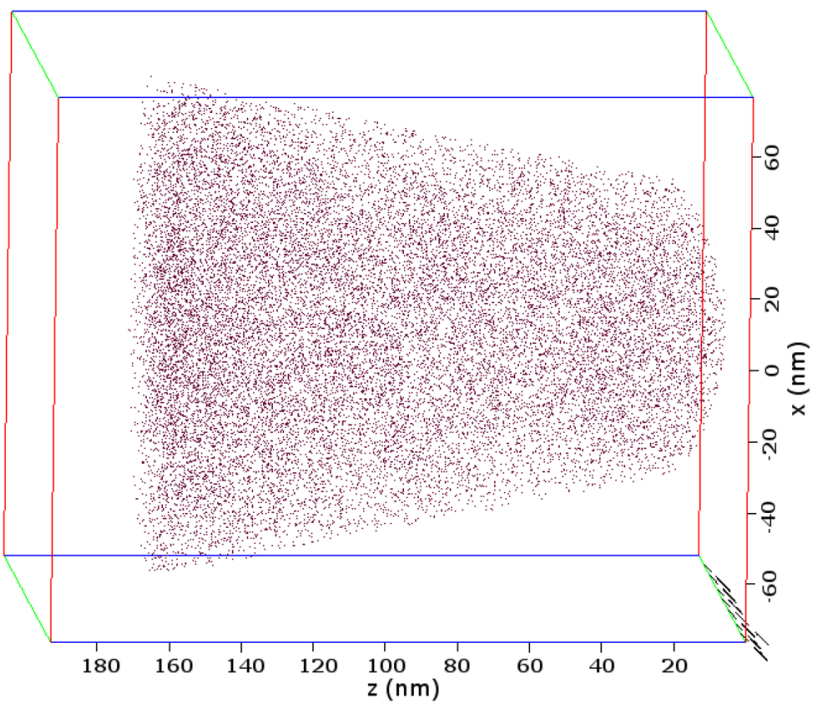

FIG. 3. 3D spatial distribution of carbon impurities in a GaAs crystal prepared by FIB in the form of a very sharp tip. 
The second important information is the high concentration of carbon impurities $(0.2 \%)$ within the crystals. The concentration corresponding to $8.8 \times 10^{19} \mathrm{~cm}^{-3}$ is at the higher end of reported values in the literature. ${ }^{14,15}$ This incorporation would render the material metallic if all carbon atoms were electrically active. This is useful for specific applications such as non-alloyed ohmic contacts or tunnel junctions but is inhibitory for the majority of optoelectronic applications. As a result, complementary analysis of dopant activity is required in order to ascertain the suitability of the material for the applications envisaged.

As mentioned in the introduction, several authors describe important carbon incorporations within their epitaxial GaAs layer grown with TMGa. Indeed, the use of gaseous organometallic sources for the element III may lead to high doping level depending on growth conditions in GaAs. One study conducted by Abernathy et al. showed that the TMGa precursor was accountable for the high carbon density within the GaAs grown layer and that the use of alternative sources as Triethygallium (TEGa) solves the problem. ${ }^{10,11}$ This different behavior can be explained by the decomposition process of the TMGa molecule. In CBE conditions, after injection in the growth chamber under vacuum $\left(10^{-2}\right.$ Torr $)$, molecules condense on the wafer surface. Then, due to the temperature imposed by a heating system, these latter will lose their hydrocarbon radicals by thermal decomposition, while the remaining semiconductor elements on the surface will contribute to the epitaxy. However, in the TMGa case, it is assumed that due to incomplete decomposition, a fraction of carbon atoms bonded to the $\mathrm{Ga}$ in the form of $\mathrm{GaCH}_{3}$ may be buried in the growing film. ${ }^{16}$

As a matter of fact, it is well known that decomposition of TMGa occurs sequentially. The kinetic studies tend to prove that the split-off energy around $250 \mathrm{~kJ} / \mathrm{mol}$ and $150 \mathrm{~kJ} / \mathrm{mol}$ for, respectively, the first and second $\mathrm{CH}_{3}$ radicals rises to $325-340 \mathrm{~kJ} / \mathrm{mol}$ for the last one. ${ }^{16,17}$ These values are accountable to the high carbon incorporation level measured by several authors. The low temperature and V/III precursor ratio used in this work could explain the $0.2 \%$ carbon incorporation measured within the GaAs microcrystals.

Moreover, it is interesting to note that hydrogen from the remaining $\mathrm{GaCH}_{3}$ ligands also has also a strong $\mathrm{H}-\mathrm{C}$ binding energy $(410 \mathrm{~kJ} / \mathrm{mol})$ and is incorporated at the same time. $^{12}$ The hydrogen passivation property of acceptor impurities (and donors) in GaAs may induce an effective free carrier concentration lower than carbon level. Otherwise, $\mathrm{C}$ atoms being a IV element can dope the III-V semiconductors such as GaAs both $\mathrm{p}$ or $\mathrm{n}$ depending on growth conditions and its position in the lattice crystal. Thereby, a doping compensation effect can also be observed. ${ }^{18}$ Consequently, the next analysis will focus on measuring the effective carrier concentration.

\section{B. Raman spectroscopy}

A possible way to determine the carrier concentration in polar semiconductors as GaAs is using Raman spectroscopy. The typical Raman spectrum of GaAs consists of two optical modes, the longitudinal optic (LO) at $292 \mathrm{~cm}^{-1}$ and the

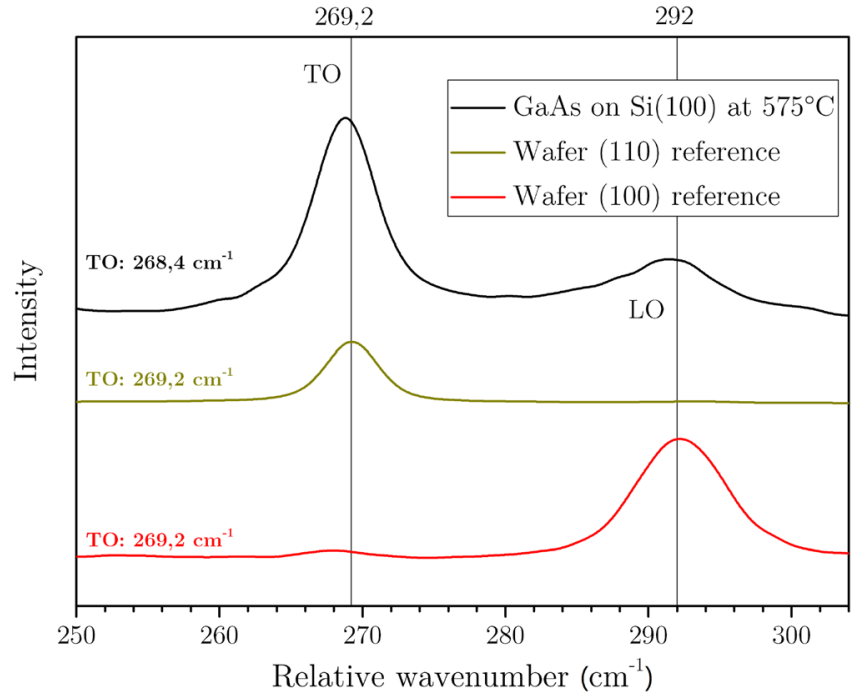

FIG. 4. Room temperature Raman spectrum of the GaAs crystal grown on $\mathrm{Si}(100)$ at $575^{\circ} \mathrm{C}$ compared with those of two GaAs wafers (100) and (110).

transverse optic (TO) at $268 \mathrm{~cm}^{-1}$ (Fig. 4). In our case, only the TO phonon is theoretically allowed whereas the LO is forbidden due to selection rules. ${ }^{19}$ The observation of one or the other modes depends on the scattering geometry. Another phenomenon takes place for doped polar semiconductors. The interaction between the collective oscillation of free carriers (plasmons) with the LO phonons creates an additional mode called the LO-plasmon coupled (LOPC) mode. As the carrier density increases at high $\mathrm{p}$ doping, the vibration frequency of this latter shifts toward lower wavenumbers. ${ }^{20}$ This makes it possible to estimate the doping level from the peak position.

In Figure 5, we show data from Seon et al. ${ }^{20}$ revealing the relationship between the LOPC frequency redshift and the hole concentration in $\mathrm{p}$ doped GaAs for different impurities. As it can be seen, this redshift depends also on the dopant size. It is interpreted by saying that both carbon and

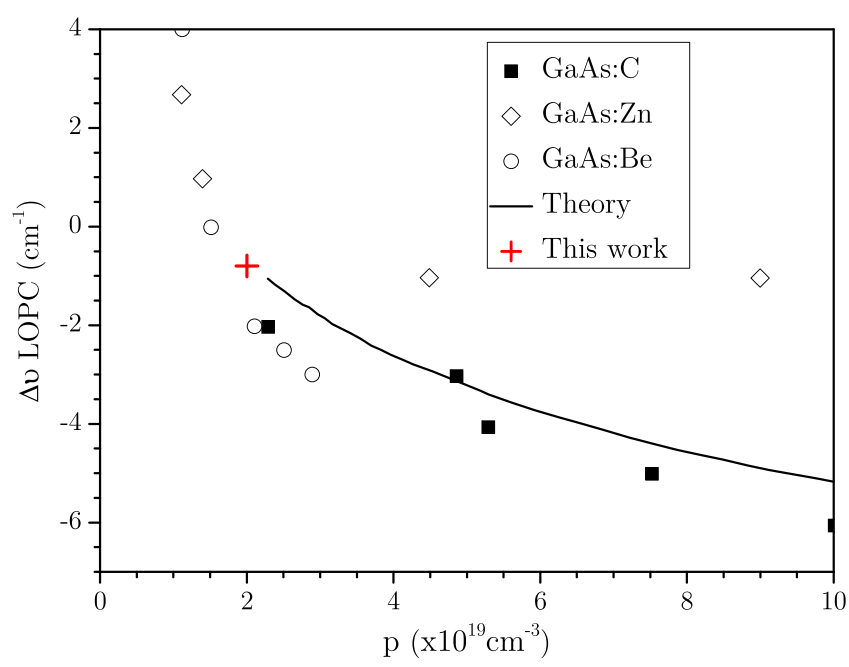

FIG. 5. Change between LOPC mode energy and that of bulk TO phonon $\left(\Delta \nu\right.$ LOPC) vs hole concentration. Data from Fukusawa et al. ${ }^{21}$ are represented by $\bigcirc$, from Irmer $\mathrm{et} \mathrm{al} .{ }^{22}$ by $\diamond$ and from Seon et al. ${ }^{20}$ by $\boldsymbol{\square}$. The solid curve is calculated by Seon et al. and was used to estimate the carrier concentration in our GaAs crystals. 
TABLE I. Extrapolated carrier concentration from LOPC redshift.

\begin{tabular}{lcc}
\hline Growth condition & $\begin{array}{c}\text { LOPC redshift } \\
\left(\mathrm{cm}^{-1}\right)\end{array}$ & $\begin{array}{c}\text { Estimated carrier } \\
\text { concentration }\left(\mathrm{cm}^{-3}\right)\end{array}$ \\
\hline on $\mathrm{Si}(100)$ at $575^{\circ} \mathrm{C}$ & -0.8 & $2 \times 10^{19}$ \\
\hline
\end{tabular}

beryllium, which are relatively small atoms with respect to gallium and arsenic, influence the vibrational spectrum of GaAs in the same manner by their substitutional position in the lattice. We can conclude two points: first, our GaAs crystal seems to have hole concentration around $2 \times 10^{19} \mathrm{~cm}^{-3}$ (Table I). Second, the high redshift is consistent with an atom of the size of carbon, which is compatible with the atomic probe tomography analysis, but seems to indicate that most of the incorporated carbons are in interstitial position.

However, regarding the difference in terms of materials and analysis, the extrapolation of a curve from another study to our results may induce errors on the estimated doping level. This method may then only served as a first indication on the nature of impurities and on the concentration of substitutional atoms.

\section{Micro-photoluminescence}

Another suitable technique for the determination of doping level is photoluminescence spectroscopy. This nondestructive and contactless method, commonly performed at room temperature, yields information on the electronic structure of materials. Basically, from the photoluminescence response, it is possible to evaluate the material quality, the bandgap, and detect defect levels in this latter. The carrier concentration is usually deduced either by comparison to calibrated samples or by referring to theoretical equation taking into account the energy shift of PL peak depending on the doping. However, the PL signal results of a complex combination of several phenomena complicate its interpretation. Furthermore, in the case of specific microstructure, such as the studied GaAs microcrystal here, no reference exists for comparison.

We have then developed a method allowing us to determine the carrier concentration by studying the PL peak energy variation of the GaAs crystals versus the temperature. This latter consists in measuring the bandgap by the PL peak centroid for a wide range of temperatures below $300 \mathrm{~K}$ and matching a theoretical equation with the carrier concentration as unknown parameter. The advantage is to greatly improve the accuracy thanks to multiple measurements.

The variation of the intrinsic bandgap energy with temperature can be expressed by the empirical Varshni equation ${ }^{23}$

$$
E_{g}(T)=E_{g}(0)-\frac{\alpha T^{2}}{T+\beta},
$$

where $E_{g}(T)$ is the bandgap energy at $T, E_{g}(0)$ is the bandgap energy of pure GaAs at $T=0 \mathrm{~K}$ equal to 1,519 $\pm 0,001 \mathrm{eV}$, and $\alpha$ and $\beta$ are empirical parameters obtained from the fit of the right hand side to experimental set of data. Here, those extracted by Olego et al. ${ }^{24}(\alpha=5.1$ $\times 10^{-4} \mathrm{eV} \mathrm{K}^{-1}$ and $\left.\beta=250 \mathrm{~K}\right)$ are in good agreement with our data.
The bandgap narrowing in heavily doped $\mathrm{P}$ and $\mathrm{N}$ type of several III-V semiconductors including GaAs can be expressed by

$$
\Delta E_{B G N}=A p^{1 / 3}+B p^{1 / 4}+C p^{1 / 2},
$$

where $p$ is the majority carrier concentration, $A, B$, and $C$ have the following constant values: $A=9,83 \times 10^{-9} \mathrm{eV} \cdot \mathrm{cm}$, $B=3,90 \times 10^{-7} \mathrm{eV} \cdot \mathrm{cm}^{3 / 4}, C=3,90 \times 10^{-12} \mathrm{eV} \cdot \mathrm{cm}^{3 / 2}$ for GaAs P type (values from Jain et al ${ }^{25}$ ) if $E_{B G N}$ is expressed in $\mathrm{eV}$ and $\mathrm{p}$ in $\mathrm{cm}^{-3}$. Combining these two models in the following expression

$$
E_{g}(T)=E_{g}(0)-\Delta E_{B G N}-\frac{\alpha T^{2}}{T+\beta} .
$$

We can fit our data with the method of least squares and thereby obtain an estimate of the majority carrier concentration (Fig. 6). It is shown that the bandgap evolution with temperature of the GaAs $\mathrm{p}(\mathrm{Zn})$ reference wafer is well fitted with this model without any change in the parameters of the literature. ${ }^{25}$ The best fit (not shown in Fig. 6 for clarity) gives a hole density of $3 \times 10^{18} \mathrm{~cm}^{-3}$. This value is well within the doping range specified by the wafer constructor data sheet. It is also close to the one derived from a four probes measurement. Being confident in this modelization of the bandgap evolution with temperature extracted from the PL(T) measurements, we then use it to derive the doping concentration of our GaAs crystals from the PL(T) measurements, and we obtained a hole concentration of $1 \times 10^{19} \mathrm{~cm}^{-3}\left(\mathrm{R}_{a d j}^{2}=0.985\right)$. To give an idea of the sensitivity of the method, we include on the graph simulations for $\mathrm{P}$ type GaAs doped at $5 \times 10^{18} \mathrm{~cm}^{-3}$ and $2 \times 10^{19} \mathrm{~cm}^{-3}$. As it can be seen, the modelization is very sensitive to a factor two variation of the doping concentration. Then, the doping concentration of our GaAs microcrystals is derived with a good accuracy from the PL(T) measurements.

The PL(T) measurements then show that the doping concentration is significantly lower than the total carbon

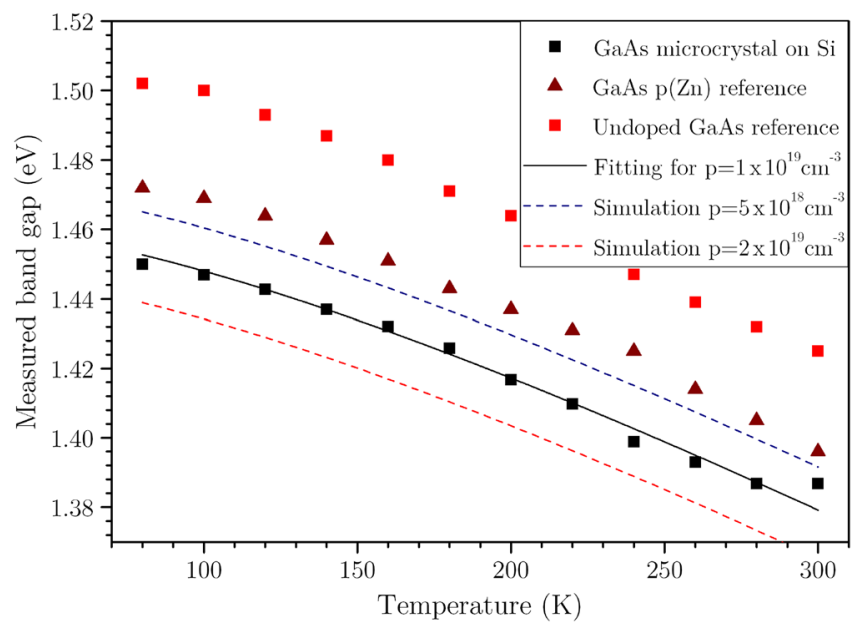

FIG. 6. Measured bandgap derived from PL as a function of temperature for a GaAs microcrystal on $\mathrm{Si}(\boldsymbol{\square})$, for a p-doped GaAs wafer (brown triangle), and for an undoped GaAs reference wafer (red square). The solid line is Eq. (3) fitted to the GaAs microcrystal on Si. The dotted lines are displayed to show the sensitivity of the simulation with Eq. (3) to a factor two variation of the dopant concentration around the best fit of the GaAs microcrystals on the Si data. 


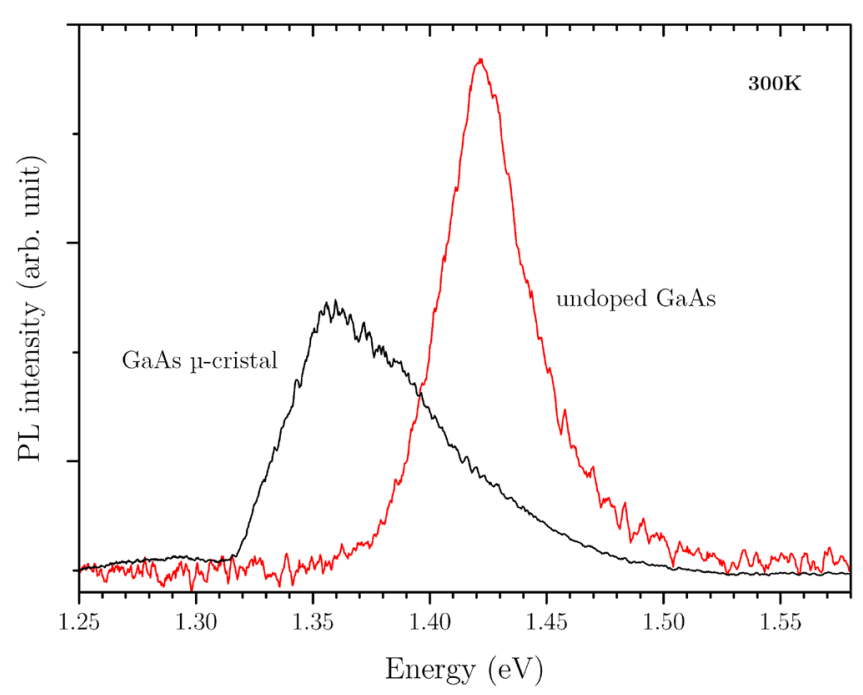

FIG. 7. Room-temperature $\mu$-PL spectra of a GaAs microcrystal grown on $\mathrm{Si}$ and of an undoped reference GaAs wafer.

concentration derived from APT measurements expressing that only a fraction close to $10 \%$ of carbon atoms are effectively active. Indeed, it is often observed in carbon doped GaAs that not all carbon atoms are electrically active, and the effective hole concentration is substantially lower than the carbon concentration. ${ }^{12,26}$ Furthermore, the higher the carbon concentration, the higher the fraction of passivated carbon. ${ }^{27}$ The same phenomenon occurs for the compensation effect. Therefore, such a difference is not unusual and could be explained by more specific chemical analyses on the position of carbon in the lattice and the possible formation of passivation complex as $\mathrm{C}-\mathrm{H}_{x}$.

Other information can also be extracted from the PL spectrum. Fig. 7 shows the PL emission of a GaAs microcrystal, as well as an undoped reference GaAs wafer for comparison. Both spectra were obtained by using the same experimental configuration, and in particular, a long range $20 \times$ infrared lens needed for PL(T) analysis with a cryostat. This limits the spot size to $5 \mu \mathrm{m}$, which is larger than a unique crystal. The measured PL intensity coming from the GaAs crystal $(1-2 \mu \mathrm{m})$ is then lowered, and the comparison with the signal of a large and planar reference GaAs wafer is difficult. Despite this fact, the integrated PL peak intensity of the GaAs microcrystal is relatively high and close to the intrinsic wafer (20\% lower), indicating a good crystalline quality as shown in previous reports. ${ }^{13,19}$ It is also interesting to note the larger full width at half maximum (FWHM) of the GaAs crystal compared to the undoped wafer, respectively, $70 \mathrm{meV}$ and $36 \mathrm{meV}$. This kind of broadening is indeed frequently reported for heavily p-type carbon doping of GaAs, which is consistent with the previous results, and

TABLE II. Carbon concentration and estimated doping level.

\begin{tabular}{lccc}
\hline \hline & APT & Raman & $\mu$-PL(T) \\
\hline C density $\left(\mathrm{cm}^{-3}\right)$ & $8.8 \times 10^{19}$ & & \\
Carrier concentration $\left(\mathrm{cm}^{-3}\right)$ & & $2 \times 10^{19}$ & $1 \times 10^{19}$ \\
\hline \hline
\end{tabular}

may be attributed to the convolution of the band-to-acceptor and band-to-band emission peaks. ${ }^{28}$

\section{CONCLUSION}

We investigated the presence and role of carbon impurities within GaAs microcrystals grown on the silicon substrate by a new selective growth method called ELTOn (Epitaxial Lateral overgrowth on Tunnel Oxide from nanoseeds) using chemical beam epitaxy with trimethylgallium as the $\mathrm{Ga}$ gaseous precursor (Table II). The electrically active concentration of carbon acting as a p-type dopant was inferred from photoluminescence versus temperature measurements and found equal to $1 \times 10^{19} \mathrm{~cm}^{-3}$. This atomic density is around $10 \%$ of the total carbon concentration derived from atomic probe tomography. These values are consistent with reports in the literature for homoepitaxial GaAs using trimethygallium as a gaseous precursor, as this molecule is known for potentially leading to high carbon concentration depending on growth condition caused by an incomplete thermal decomposition. Finally, the methodology we have developed is convenient and accurate, enabling the determination of type of doping and carrier concentration within micrometric GaAs crystals directly integrated on silicon substrates. The optimization of GaAs devices integrated on silicon substrates is now underway to take full advantage of both family materials for optoelectronic applications.

\section{ACKNOWLEDGMENTS}

This work was supported by the ANR (project MULTISOLSI No. 2011 PRGE 00901). The authors also thank the Centrale de Technologie Universitaire MINERVE and RENATECH for technological backup.

${ }^{1}$ Y. Q. Wu, M. Xu, P. D. Ye, Z. Cheng, J. Li, J.-S. Park, J. Hydrick, J. Bai, M. Carroll, J. G. Fiorenza, and A. Lochtefeld, Appl. Phys. Lett. 93, 242106 (2008).

${ }^{2}$ K. L. Lew, S. F. Yoon, W. K. Loke, H. Tanoto, C. L. Dohrman, D. M. Isaacson, and E. A. Fitzgerald, J. Vac. Sci. Technol., B 25, 902 (2007).

${ }^{3}$ K. Tanabe, K. Watanabe, and Y. Arakawa, Sci. Rep. 2, 349 (2012).

${ }^{4}$ Y. Chriqui, G. Saint-Girons, G. Isella, H. v. Kaenel, S. Bouchoule, and I. Sagnes, Opt. Mater. 27, 846 (2005).

${ }^{5}$ T. Maruyama, T. Okumura, S. Sakamoto, K. Miura, Y. Nishimoto, and S. Arai, Opt. Express 14, 8184 (2006).

${ }^{6}$ M. R. Lueck, C. L. Andre, A. J. Pitera, M. L. Lee, E. A. Fitzgerald, and S. A. Ringel, IEEE Electron Device Lett. 27, 142 (2006).

${ }^{7}$ J. M. Zahler, K. Tanabe, C. Ladous, T. Pinnington, F. D. Newman, and H. A. Atwater, Appl. Phys. Lett. 91, 012108 (2007).

${ }^{8}$ S. F. Fang, K. Adomi, S. Iyer, H. Morko, H. Zabel, C. Choi, and N. Otsuka, J. Appl. Phys. 68, R31 (1990).

${ }^{9}$ C. Renard, T. Molière, N. Cherkashin, J. Alvarez, L. Vincent, A. Jaffré, G. Hallais, J. P. Connolly, D. Mencaraglia, and D. Bouchier, Sci. Rep. 6, 25328 (2016).

${ }^{10}$ C. R. Abernathy, S. J. Pearton, F. Ren, W. S. Hobson, and P. W. Wisk, J. Vac. Sci. Technol., A 12, 1186 (1994).

${ }^{11}$ C. Abernathy and P. Wisk, Appl. Phys. Lett. 60, 2421 (1992).

${ }^{12}$ B. Lamare, J. L. Benchimol, M. Juhel, B. Akamatsu, P. Legay, and F. Alexandre, J. Cryst. Growth 141, 347 (1994).

${ }^{13}$ C. Renard, N. Cherkashin, A. Jaffré, L. Vincent, A. Michel, T. Molière, R. Hamouche, V. Yam, J. Alvarez, F. Fossard et al., Appl. Phys. Lett. 102, 191915 (2013).

${ }^{14}$ C. R. Abernathy, S. J. Pearton, R. Caruso, F. Ren, and J. Kovalchik, Appl. Phys. Lett. 55, 1750 (1989).

${ }^{15}$ M. Konagai, T. Yamada, T. Akatsuka, K. Saito, E. Tokumitsu, and K. Takahashi, J. Cryst. Growth 98, 167 (1989). 
${ }^{16}$ N. Pütz, H. Heinecke, M. Heyen, P. Balk, M. Weyers, and H. Lth, J. Cryst. Growth 74, 292 (1986).

${ }^{17}$ M. G. Jacko and S. J. W. Price, Can. J. Chem. 41, 1560 (1963).

${ }^{18}$ T. M. Schmidt, P. P. M. Venezuela, M. J. Caldas, and A. Fazzio, Appl. Phys. Lett. 66, 2715 (1995)

${ }^{19}$ C. Renard, N. Cherkashin, A. Jaffre, T. Molière, G. Hallais, L. Vincent, J. Alvarez, D. Mencaraglia, A. Michel, and D. Bouchier, J. Cryst. Growth 401, 554 (2014).

${ }^{20}$ M. Seon, M. Holtz, W. M. Duncan, and T. S. Kim, J. Appl. Phys. 85, 7224 (1999).

${ }^{21}$ R. Fukasawa, K. Sakai, and S. Perkowitz, Jpn. J. Appl. Phys., Part 1 36, 5543 (1997).
${ }^{22}$ G. Irmer, M. Wenzel, and J. Monecke, Phys. Rev. B 56, 9524 (1997).

${ }^{23}$ Y. P. Varshni, Physica 34, 149 (1967).

${ }^{24}$ D. Olego and M. Cardona, Phys. Rev. B 22, 886 (1980).

${ }^{25}$ S. C. Jain, J. M. McGregor, and D. J. Roulston, J. Appl. Phys. 68, 3747 (1990).

${ }^{26}$ D. M. Kozuch, M. Stavola, S. J. Pearton, C. R. Abernathy, and J. Lopata, Appl. Phys. Lett. 57, 2561 (1990).

${ }^{27}$ S. A. Stockman, A. W. Hanson, S. M. Lichtenthal, M. T. Fresina, G. E. Hfler, K. C. Hsieh, and G. E. Stillman, J. Electron. Mater. 21, 1111 (1992).

${ }^{28}$ H. D. Chen, M. S. Feng, P. A. Chen, K. C. Lin, and J. W. Wu, Jpn. J. Appl. Phys., Part 1 33, 1920 (1994). 